\title{
Fundamental Physical Constant
}

National Cancer Institute

\section{Source}

National Cancer Institute. Fundamental Physical Constant. NCI Thesaurus. Code C67361.

A physical quantity that is constant in all circumstances throughout the universe (e.g., speed of light c, elementary charge, the gravitational constant G). 